\title{
Mind as an Important Environmental Priority and the Need for Ecologically-targeted Thinking
}

\author{
Tatiana Leshkevich \\ Southern Federal University, Russia \\ 105/42 Bolshaya Sadovaya Str. \\ Rostov-on-Don, Russian Federation 344006 \\ E-mail: Leshkevicht@mail.ru
}

\begin{abstract}
The article substantiates the importance of environmental priorities and ecologically-targeted thinking in the era of modern technogenic development. The goal is to justify the fact that the quality of environmental interactions depends on the degree of human reasonableness. Developing this idea, the author analyzes the arguments of Russian scientists N. Timofeev-Resovsky, V. Vernadsky, V. DanilovDaniljan, N. Moiseev and V. Stepin. The methodological novelty of the research is related to using the potential of postnon-classical methodology. The author uses a scenario approach related to the modeling of the future. Of paramount importance is using data banks in order to model dynamic processes in development. The article concludes that there is a need for an environmental paradigm, which is accompanied by an ideology of conscious regulation and brings a new type of responsibility to light.
\end{abstract}

Keywords-mind; ecological thinking; environmental priorities; post-non-classical methodology; uncertainty

\section{INTRODUCTION}

The modern era defines itself as a "non-equilibrium, unstable" world with rapidly developing technologies, multiple risks and threats. "Blindly" developed technologies, inherent in uncontrollable negative consequences, happen to be in-built into fundamental interrelations between the social and the natural. It is true that nature is in continuous need for regeneration and every organism, including the social one, is integrated into its environment. At the same time, increasing pressures on ecosystems lead to serious environmental deformations. The need for an environmental assessment of technological development and its impact on human life arises. There is an obvious necessity to radically relieve the pressure of man-induced influence on ecosphere.

The main idea of our research is to assert that the quality of environmental interactions is highly dependent on the degree of human reasonableness. Awareness of this fact causes more stringent requirements for technological development and demands such technologies which would be directed to achievement of adaptation effect and would not harm nature. Therefore, the reasonable regulation of natural and socio-anthropic interactions must be recognized as a strategically significant priority. In other words, the "new order of reality" presupposes rational control over natural, technical and socio-anthropic interactions.

\section{II. "PARTING WITH SimPlicity" AND ECOLOGICALLY -} TARGETED THINKING: ARGUMENTS OF RUSSIAN SCIENTISTS

Despite the fact that the problems of ecology have always been at the center of the research interest of many scientists, few of the researchers linked them directly to the human mind. As a rule, objective factors were the main objects of research. So, the famous Russian scientist V. I. DanilovDanilyan questioned the acceptability of replacing the mechanisms of natural resistance with technological tools The scholar's conclusion consisted in the assertion that, environment is a united whole, environmental deterioration inevitably leads to disastrous consequences. In this case, the evaluation procedure with regard to ecological situation on a global scale is extremely vital as viewed by V. I. DanilovDanilyan[1]. However, it should be noted that the previously an established Russian scholar N. V. Timofeev-Resovsky expressed serious concern about a certain environmental norm stating the fact that «at the end of the day, people can't live on the Planet with no or dysfunctional ecosphere» [2].

In this context, the position of another Russian scientist N.N. Moiseev seemed particularly interesting. N. Moiseev justified the right of the future generations to be in possession of resources and environmental conditions equivalent to those in the possession of the living [3]. The book, written by N. Moiseev, «Parting with simplicity» can be viewed as a rationale for a strategy for future development whose goal is to achieve harmonization of external and internal ecology [4]. As he believed, this issue should disturb not only the minds of scientists, but of all mankind. According to N. Moiseev, «the concept of nature» should be formulated in a way which will make it possible for a Man to provide for his own future, relying on this concept. The author considered the outgoing century to be "the age of warning" [4].

Presumably, N. Moiseyev largely relied on the findings of the outstanding Russian scientist V.I. Vernadsky, whose doctrine of the "noosphere" plays a huge role in shaping the ecological worldview. It was V. I. Vernadsky who paid particular attention to the fact that Man is "one of geological forces among others", "his thoughts and doings" should be 
viewed as a dynamic systemically important factor capable of generating "a new order of reality" [5].

In this regard, the following principal conditions should be noted. Firstly, the investigation of convergence of external and internal environmental factors cannot be reduced to social, economic, technical or biological circumstances taken separately. Secondly, the concept of biotic regulation of the environment indicates that the biota not only passively adapts to environmental factors, but also has a regulatory effect on them. Biota, in accordance with the conclusions of modern science, can include compensatory mechanisms, retain threshold characteristics and suppress disturbances. Thirdly, the modern environmental approach should be focused on the foundations of rational humannatural relations, taking into account concrete facts and figures.

It is true, that in the conditions of the global world and a Great science, it is possible to create data banks and model processes in integrity, using computer simulation techniques. In this connection, it can be assumed that modern science is capable of setting up a system of prohibitions and formulating an ecological imperative, which would guarantee the stability of the human development. However, there arises a question of whether mankind will be able to accept and adopt restrictions set by science? This critical question is addressed to the mind of mankind and underscores the need for the development of global environmental ethics and the creation of continuous environmental education system.

At the same time, the modern researchers take into account the following circumstances: "1) an increased challenging of scientific authority, 2) the need for interdisciplinary to cope with ever more complex problems and 3) to elaborate on a social epistemology [6]. These comments seem reasonable. However, from our point of view, the basic foundations of environmental relations should be a system of humanitarian values and priorities of the humanitarian sphere. What kind of future we want? What is the image of the desired future? The humanity scholars are sure that development strategies should be targeted at implementing genuinely humanistic values. It is necessary to realize the full measure of responsibility and the desire to striving to prevent forthcoming crises. With certainty, we can conclude that the project world-models should be based on genuine human values. The ideology of environmentally effective human behavior should be scientifically grounded in opposition to its consumer behavior.

The well-known Russian philosopher V. S. Stepin assumes to consider a new aspect of this problem. He stresses that "culture preserves, translates and generates the programs of activity, behavior and cognition which consist of the aggregate social-historical experience [7]. According to this position, the central core is a culture that generates programs of human activity. In this case, it is reasonable to assume that culture is responsible for the ecological restructuring of thinking and consciousness. Therefore, identifying environmental priorities and potential development scenarios involves an insight into human activity as an essential geopolitical force.

But first of all it would be necessary to pay attention to two opposite processes. On the one hand, the impact of human activity on the environment is rapidly increasing. On the other hand, human being in many respects depends on changes in the ecosphere. In fact, both adaptation to the environment and adaptation of the environment itself to human needs are essential. At the same time, nowadays it seems obvious that the development of the modern world has made a rapid technological turn, it is closely connected with the modern technological civilization, in which the relations of man-nature go to the background. Modern technologies, penetrating into all spheres of life, try to prove that ecodependence as a fundamental dimension of life is not insurmountable. Thereby the mankind is involved in development of scenarios of the technological future. From the point of view of our approach, the development prospects have to be connected with environmental priorities. As the person and the nature are bound together inseparably, it is important that both people's thinking and behavior become environmentally oriented. We believe that the human mind should show its practical power and give humanity a chance to harmoniously solve the acute problem of "eco-dependence - eco-independence".

\section{INNOVATIVE METHODOLOGICAL APPROACHES TO THE RESEARCH}

The problem of studying environmental priorities involves the use of innovative methodological tools. From our point of view, the methodological novelty of the conducted research is related to using the potential of postnon-classical methodology, especially the following interrelated methodological guidelines. Firstly, the priority position is the implementation of the scenario approach. It is associated with modeling of the future and must be based on a system of universal values. Its obvious advantages, in our opinion, are connected both with the transfer of actual environmental characteristics, and with a specific choice of behavioral patterns. Secondly, according to the conducted research, the principal basis is the application of the principle of integrity, which is the important methodological tool and allows us to correlate external and internal environmental factors. Thirdly, this research highlights the significance of the synergetic methodology and, in particular, to the synergetic principle of recursive interaction of nature and artifacts. It is true that nature is in continuous need for regeneration. Awareness of this fact causes strict requirements for technological development and demands such technologies which would be directed to achievement of adaptation effect. Fourthly, discussing the innovative methodological strategy, one should to point out the significance of both the systems and interdisciplinary approaches. Of paramount importance is using data banks in order to model dynamic processes in development and referring to sociological, theoretical and concept-based material.

These methodological approaches are of particular value, due to the fact that they contribute to summarizing the 
current data of various disciplinary areas in an integral scientific theory. Using these methodological tools, scientists could not only determine the existing ecological state of the external and internal environment of the social system. An innovative methodological strategy makes it possible to construct a generalized development model. It should be noted that in the current situation, the convergence strategy is assessed as a global perspective of the Big Science. It facilitates decision-making process in the conditions of uncertainty. It is not surprising that the concepts of "uncertainty" and "complexity" are key concepts of modern post-non-classical methodology [8]. The scenarios of the future are connected with the acceptance of uncertainty, instability, alternativeness, the social entropy and risks which have already become habitual components of the everyday existence. Scholars and especially methodologists focus on studying the "causes of spontaneous structure genesis". At the same time, it becomes clear that the purpose of the scientific forecast is to avoid the large-scale negative consequences of global technological development and environmental disasters.

In addition to the highlighted methodological approaches, we also see the effectiveness of such methodological tools as the principle of "specificity", the comparative analysis and the levels of detail. Methodologists are sure that the environmental dimension allows us to localize each event in space and time. This provides an opportunity to study a particular phenomenon in the specific circumstances and conditions of its functioning. Thus, an innovative methodological strategy is a vital part of the reflexive philosophical analysis of the environmental transformations of the modern era. Apparently, this could become a common platform that provides both the convergence process of natural and humanitarian knowledge, as well as the rational regulation ideology, aimed at achieving eco-independence. Apparently, it could become a common platform that provides both the convergence process of natural-science and humanitarian knowledge, as well as the rational regulation ideology, aimed at achieving of functional eco-independence.

\section{CONCLUSION}

Developing the idea of mind as the most important environmental priority, we would like to draw attention to the following conclusions. In contrast to rapid technological development, this analysis points to the need to form an environmental paradigm, which brings a new type of responsibility to light. Every person is responsible for the organization of the relations with the nature, society and other people. The main principle is that both internal and external ecology should be correlated with genuinely humanistic programs of activity.

As a result, the ecological worldview becomes an urgent requirement of the present, especially in the conditions of rapid technological development. It promotes the generation of human activity programs in many respects different from consumer ones. It is not only the interaction between nature and society that ecological outlook is applicable to. According to the generally accepted position, it is universal and is aimed at studying the ecology of the community, family ecology, the ecology of the personality, the ecology of professional activity, ecology of everyday life and so on. All human life passes in certain ecological conditions and a person cannot be indifferent to the environment of his dwelling. It is quite obvious that the disagreement of an artificial environment leads to problems of both valeological and psychological, and cognitive courses. As rightly emphasized by F.Varela, E. Thompson, and E. Rosch, Man seems to be a part of this global convergence and interrelated co-genesis[9]. Environmental compatibility of man and the world is connected with the principle: «Primum non nocere!»

Probably, therefore, ecological ethics is recognized as an important educational discipline, focusing on the moral relations of man and his environment. In the light of all the unsolved global problems of modernity, the process of education has to be constructed on the paradigm of ecology, accompanied by the ideology of conscious regulation of human-nature relations, taken in all their diversity. The history of human development has shown that the ecological situation has always been an important factor in social and historical events and achievements. Due to the fact that neither man nor society can be full freed from natural connections, the ecological dimension should be evaluated as the fundamental basis of human life. It seems reasonable that the environmental paradigm, accompanied by the ideology of conscious regulation, should be considered as the main priority of modern development.

\section{REFERENCES}

[1] V. Danilov-Danilyan, "The Evaluation of Engineering and Economic Operations from the Perspective of Global Ecology", in Philosophy of Science and Technology - Nature and Technology at the Turn of the Third Millennium. Moscow: Rossijskoe Filosofskoe Obshchestvo, 2005, pp. 44-57.

[2] N. Timofeeff-Ressovsky, "Biosphere and Mankind", in Scholarly Works by Obninsk Departmant of USSR Geographic Society. Obninsk, 1968, 1(1), pp. 3-12.

[3] N. Moiseev, "The Rise to Reason". Moscow: IzdAT, 1993.

[4] N. Moiseev, "Parting with Simplicity", Moscow: Agraf, 1998, p.7.

[5] V. Vernadsky, "Scientific Thought as a Planetary Phenomenon". Moscow: Nauka, p.132.

[6] A. Ruser, "Perspectives of and Challenges for a Social Philosophy of Science: Highlighting the Challenges", in Epistemology \& Philosophy of Science. Moscow: KNORUS. T. XLV, N 3, pp.54-64.

[7] V. Stepin, "Consrtuctive and prognostic function of philosophy" in XXII World Congress of Philosophy. Rethinking philosophy today. Seoul: Seoul National University, 2008, p.29.

[8] T. Leshkevich, "Vector for Modifying the Methodology in the Direction of Convergence Socio-humanitarian Knowledge with Postnon-classics", in Bulletin of the Tomsk State University. Philology, 2018, № 427, pp.71-78

[9] F. Varela, E. Thompson, and E. Rosch, "The Embodied Mind. Cognitive Science and Human Experience". Cambridge, MA: The MIT Press, 1991, p. 150. 